\title{
Risk factors and their interaction on chronic kidney disease: A multi-centre case control study in Taiwan
}

Sui-Lung Su${ }^{1}$, Chin Lin ${ }^{2}$, SenYeong Kao ${ }^{1}$, Chia-Chao Wu ${ }^{3}$, Kuo-Cheng Lu' ${ }^{4}$, Ching-Huang Lai ${ }^{1}$, Hsin-Yi Yang ${ }^{1}$, Yu-Lung Chiu', Jin-Shuen Chen ${ }^{3}$, Fung-Chang Sung ${ }^{5}$, Ying-Chin Ko ${ }^{5}$, Chien-Te Lee ${ }^{6}$, Yu Yang ${ }^{7}$, Chih-Wei Yang ${ }^{8}$, Shang-Jyh Hwang ${ }^{9}$, Ming-Cheng Wang ${ }^{10}$, Yung-Ho Hsu ${ }^{11}$, Mei-Yi Wu ${ }^{11}$, Yu-Mei Hsueh ${ }^{12}$, Hung-Yi Chiou ${ }^{12^{*}}$ and Yuh-Feng Lin Li, $^{3 *}$

\begin{abstract}
Background: Chronic kidney disease (CKD) is highly prevalent in Taiwan. More than two-thirds of end-stage renal disease is associated with diabetes mellitus (DM) or hypertension (HTN). Therefore, the formulation of a special preventative policy of CKD in these patients is essential. This study surveyed 14 traditional risk factors and identified their effects on CKD in patients with HTN/DM and compared these with their effects in the general population.

Methods: This study included 5328 cases and 5135 controls in the CKD/HTN/DM outpatient and health centres of 10 hospitals from 2008 to 2010. Fourteen common effect factors were surveyed (four demographic, five disease and five lifestyle), and their effects on CKD were tested. Significance tests were adjusted by the Bonferroni method. Results of the stratified analyses in the variables were presented with significant heterogeneity between patients with different comorbidities.

Results: Male, ageing, low income, hyperuricemia and lack of exercise habits were risk factors for CKD, and their effects in people with different comorbidities were identical. Anaemia was a risk factor, and there was an additive effect between anaemia and HTN on CKD. Patients with anaemia had a higher risk when associated with HTN [odds ratio $(\mathrm{OR})=6.75,95 \%$ confidence limit $(95 \% \mathrm{Cl})$ 4.76-9.68] but had a smaller effect in people without HTN (OR 2.83, $95 \%$ Cl 2.16-3.67). The association between hyperlipidaemia-related factors and CKD was also moderated by HTN. It was a significant risk factor in people without HTN $(\mathrm{OR}=1.67,95 \% \mathrm{Cl} 1.38-2.01)$ but not in patients with HTN (OR $=1.03,95 \%$ Cl 0.89-1.19). Hepatitis B, hepatitis C, betel nut chewing, smoking, alcohol intake and groundwater use were not associated with CKD in multivariate analysis.
\end{abstract}

Conclusions: We considered that patients with HTN and anaemia were a high CKD risk population. Physicians with anaemic patients in outpatient clinics need to recognise that patients who also have HTN might be latent CKD cases.

Keywords: Chronic kidney disease, Hypertension, Anaemia, Hyperlipidaemia, Interaction

\footnotetext{
* Correspondence: hychiou@tmu.edu.tw; linyf@s.tmu.edu.tw

${ }^{12}$ School of Public Health, Taipei Medical University, No. 250, Wuxing St.,

Xinyi District, Taipei 110, Taiwan

${ }^{3}$ Division of Nephrology, Department of Medicine, Tri-Service General

Hospital, National Defense Medical Center, Taipei, Taiwan

Full list of author information is available at the end of the article
} 


\section{Background}

Chronic kidney disease (CKD) is an important public health issue because these patients have an increased risk of end-stage renal disease (ESRD). Taiwan has a high prevalence of CKD [1] and ESRD [2]. These patients are at increased risk for cardiovascular events and progression to kidney failure [3]. The benefits of screening at-risk populations and estimating progression of CKD are well established [4]. A previous study has shown that screening people with hypertension (HTN), diabetes mellitus (DM) or age $>55$ years is the most effective strategy to detect patients with CKD [5]. Therefore, planning a specific population screening/prevention strategy for people with HTN or DM is a major public health challenge. To our knowledge, there is no systematic evidence at present to confirm that a screening/prevention strategy for the general population would apply to high risk groups.

CKD is a complex disease that has complex aetiologies, but the effects of these factors are mild. The traditional factors that have an effect on CKD are primarily divided into three parts: demographic characteristics (gender [6], age [7], obesity [8] and social economic [9]), comorbidity [hepatitis B (HB) [10], hepatitis C (HC) [11], hyperuricemia [7], anaemia [12] and hyperlipidaemia [7]] and lifestyles (smoking status [13], alcohol intake [14], betel nut chewing [15], exercise habits [16, 17] and groundwater use [18]). Foregoing factors have been extensively investigated and some studies have investigated their effects in populations. However, these reported effects are inconsistent in different populations. For example, obesity had a significant effect in the general population [8] but not in patients with DM [19]. This suggests that the effects of obesity on CKD may be associated with DM. In addition, several studies have investigated the interaction between some of these factors and HTN/DM on CKD. Other studies have reported an interaction between HTN and smoking [20] and between DM and hyperuricemia [21] on renal outcomes. As a result of these various reports, we suspected that the effect of each factor on CKD may be different in healthy populations and in patients with DM/HTN.

Studies have shown evidence that the effects of some factors on CKD may depend on the presence of DM or HTN, which means that some risk factors may or may not be important in patients with HTN/CKD. This information may be important for clinical decision making for CKD patients with HTN/CKD. However, to our knowledge, no study has systematically investigated the potential factors which may have a DM- or HTN-dependent effect on CKD. Therefore, the aim of this study was to investigate different targets for screening/prevention strategies between healthy people and patients with HTN or DM that may aid in planning most effective prevention strategies for patients with DM and HTN.

\section{Methods}

\section{Population and definition}

A multi-centre project in January 2008 to July 2010 was conducted to survey the risk factors for CKD in Taiwan. Fourteen hospitals equally participated in the program. Before starting the study, appropriate sample size estimation was calculated using OpenEpi (http://www.openepi.com/ SampleSize/SSCC.htm). The settings used were: a twosided test with a power of $(1-\beta)=0.95$ at a significance level of $\alpha=0.05 / 14$, the ratio of controls to cases $=1$, the hypothetical proportion of controls with exposure $=5$ and the least extreme odds ratio $(\mathrm{OR})$ to be detected $=1.5$. Based on these settings, the study sample size required was at least 9104 subjects.

To identify the specific risk factors in patients with HTN/DM and to maintain the statistical power of this study, a higher number of patients with HTN/DM were required. Based on this, we also recruited participants without CKD from HTN/DM outpatient and health centres from each hospital as the control group. The CKD cases were recruited from nephrology and HTN/DM outpatient clinics in each hospital and from health centres and were classified as the case group. Finally, a total of 12,082 participants older than 18 years were recruited consecutively in this project (Fig. 1).

The study included 11,552 (95.6\%) participants with no missing DM and HTN status information. Exclusions included 524 participants who had cancer and 565 from four hospitals because of insufficient recruitment numbers ( $<150$ participants from three hospitals and one had only 75 healthy controls). Finally, a total of 10,463 (5218 male and 5245 female) participants (median age 57.0 years) from 10 different hospitals were included in the study.

CKD was defined as an estimated glomerular filtration rate (eGFR) of $<60 \mathrm{~mL} / \mathrm{min} / 1.73 \mathrm{~m}^{2}$ or with proteinuria. The eGFR was calculated with the equation eGFR $(\mathrm{mL} /$ $\left.\min / 1.73 \mathrm{~m}^{2}\right)=186 \times \mathrm{Cr}^{-1.154} \times$ age $^{-0.203} \times 0.742$ (if female) [22]. A total of 5328 participants that met the above criteria were included in the cases group and 5135 others in the control group. The different stages of CKD and non-CKD was defined as follows: CKD Stage 1 eGFR $\geq 90$ with albumin excretion $\geq 150 \mathrm{mg}$ and microhematuria and/or renal parenchymal disease, CKD Stage 2 eGFR 60.0-89.9 with above mentioned criteria, CKD Stage 3 eGFR 30.0-59.9, CKD Stage 4 eGFR 15.0-29.9, CKD Stage 5 eGFR $\bowtie 15$, and non-CKD eGFR $\geq 60$ without above mentioned criteria.

The definition of diabetes and HTN was based on physician diagnoses using a fasting glucose level of $>126 \mathrm{mg} / \mathrm{dL}$ [23] and a systolic/diastolic blood pressure level of $>130$ / $80 \mathrm{mmHg}$ [24], respectively. The study participants were placed into four groups according to their disease status: Group I consisted of 3785 participants without both DM and HTN (1382 cases and 2403 controls), Group II 


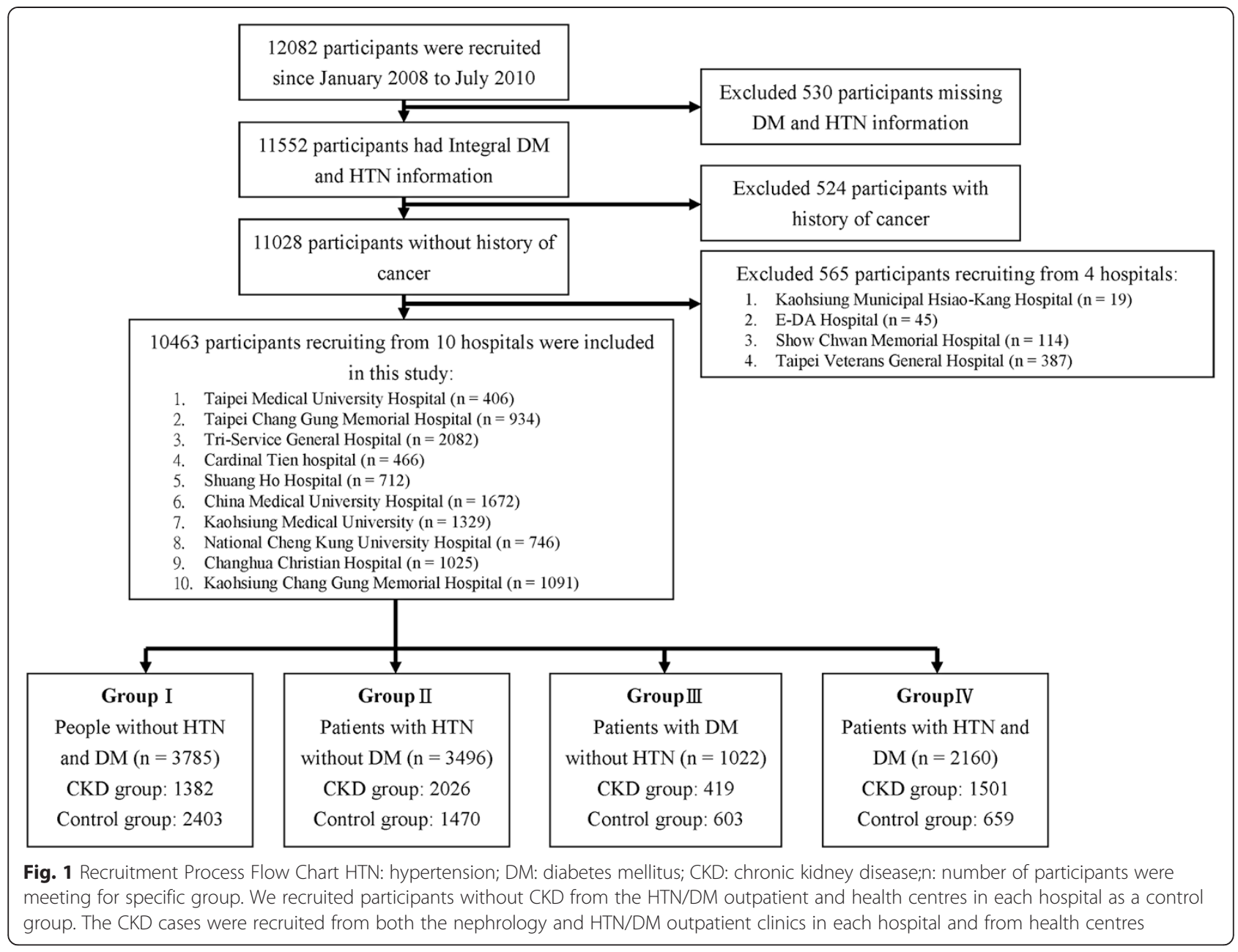

consisted of 3,496 participants with HTN and without DM (2026 cases and 1470 controls), Group III consisted of 1022 participants with DM and without HTN (419 cases and 603 controls) and Group IV consisted of 2160 participants with both DM and HTN (1501 cases and 659 controls).

\section{Risk factor assessment and definition}

Demographic characteristics (gender, age, obesity and socioeconomic), history of disease ( $\mathrm{HB}, \mathrm{HC}$, hyperuricemia, anaemia and hyperlipidaemia) and lifestyle (smoking status, alcohol intake, betel nut chewing, exercise habits and use of groundwater status) were the possible risk factors that were investigated for their individual effects and for the different effects on CKD in the four study groups. A detailed medical history, anthropometric measurements, laboratory analyses, and a health appraisal questionnaire eliciting demographic, socioeconomic and behavioural risk factors were conducted through face-to-face interviews with each participant by well-trained investigators at the initial visit. Written informed consent was obtained from all study participants.
Gender and age were assessed based on self-reporting. Body mass index (BMI) was calculated as weight $/$ height $^{2}$ $\left(\mathrm{kg} / \mathrm{m}^{2}\right)$ and participants whose BMI was $>27$ were classified as obese. The socioeconomic status was based on income and individuals were divided into three groups: low income was defined as less than 20,000 New Taiwan Dollars (NTD) per month, median income was between 20,000 and 60,000 NTD per month and high income was more than 60,000 NTD per month (1 US dollar $=30$ NTD).

Participants were recorded with a history of disease if there was an affirmative answer to having ever been diagnosed by a doctor with $\mathrm{HB}, \mathrm{HC}$, hyperuricemia, anaemia or hyperlipidaemia. The diagnosis of anaemia used in Taiwan is defined by the WHO as haemoglobin $<12 \mathrm{~g} / \mathrm{dL}$ in women and $<13 \mathrm{~g} / \mathrm{dL}$ in men [25].

Smoking status (current, former or never) was ascertained at the time of enrolment. Individuals who had not smoked more than 100 cigarettes in their lifetime were classified as never-smokers, based on common conventions in epidemiology research [26]. Others were grouped together in the smokers group. 
Alcohol intake was defined as total alcohol consumption in grams per day. We calculated this from questions about different liquors, frequencies and concentrations.

A previous study reported that moderate alcohol consumption (10-20 g/day) is associated with favourable levels of several cardiovascular risk factors [27]. Therefore, individuals who had intakes of $>20 \mathrm{~g} /$ day that continued for more than 1 month were classified in the ever-drink group, and others were classified in the never-drink group.

Betel nut chewing and exercise habits were based on a self-report questionnaire. If the participants gave a positive answer to having ever had habits of betel nut chewing or exercise, they were classified in the ever-group and the others in the never-group. Individuals who participated in physical activity fewer than three times/week were classified in the never-group [26].

Using groundwater was ascertained by the participants as having and using stable groundwater. Individuals who used groundwater for a period of more than 1 year were classified as the ever-used group and others were classified as the never-used group.

\section{Ethics statement}

The study was reviewed and approved by the institutional ethical committee of Taipei Medical University Hospital (201204035), Taipei Chang Gung Memorial Hospital (97-2187B), Tri-Service General Hospital (100-05-197), Cardinal Tien Hospital (201204035), Shuang Ho Hospital (201204035), China Medical University Hospital [DMR 101-IRB2-273 (CR-1)], Kaohsiung Medical University Chung-Ho Memorial Hospital (KMUH-IRB-20120019), National Cheng Kung University Hospital (ER-101-117), Changhua Christian Hospital (120405) and Kaohsiung Chang Gung Memorial Hospital (101-1096B). After a complete explanation of the study, written informed consent was obtained from all participants. All clinical and biological samples were collected and DNA was genotyped following patient consent.

\section{Statistical analysis}

The total cohort, including participants from different sources (HTN/DM outpatient and health centres) may have distorted the proportion of cases/controls with HTN/DM, and this could have resulted in the wrong risk assessments of HTN/DM and some of the factors relating to HTN/DM. Therefore, this study did not investigate the risk of HTN/DM on CKD, because the exposure rate of HTN/DM may be biased. The HTN/ DM cases were stratified in all analyses in order to investigate the interactions between HTN/DM and the other factors, and the stratified analyses could help reduce the confounding effect.

The significance tests were adjusted by the Bonferroni method and a $p$-value of $<0.05 / 14=0.0036$ was considered significant for avoiding the error of multiple testing. Statistical analyses were conducted with R 3.0.1 software with the 'Ime4,' 'meta' and 'metafor' package. The dependent variable for the analyses was patients with CKD. Categorical and continuous variables were presented as the number (proportion) and mean \pm standard deviation (SD). The post powers were calculated based on G*Power 3.1.7 [28].

To test the effect of each factor and to control the hospital-clustering effect (Simpson paradox), all factors were tested using hierarchical generalised linear models in the four groups (Groups I-IV). The OR and $95 \%$ confidence interval $(95 \% \mathrm{CI})$ were presented for the effects of risk factors on CKD. All variables were adjusted in multivariate analyses regardless of whether they were significant in the univariate analyses. Meta-analysis was used to quantify the difference between the coefficients of a specific variable in the different groups. The $\mathrm{I}^{2}$ test for estimating the heterogeneity between the four groups and the Cochrane Q test were used. An I ${ }^{2}>75 \%$ represented a high heterogeneity and implied that the interaction between that variable and HTN/DM may exist [29]. The $\tau^{2}$ statistic was estimated by the restricted maximum-likelihood estimator method, and a randomeffects model based on the Mantel-Haenszel method was applied. When the heterogeneity of the coefficients of a specific variable was significant in the multivariate analyses, we presented the results of the stratified analyses and attempted to find the cause of heterogeneity (DM or HTN). Moreover, the results of the pooled analyses were considered more reliable in study populations. The change of $\tau^{2}$ statistic that was estimated was used for assessment for the cause of heterogeneity.

\section{Results}

Table 1 shows the proportion of cases and controls with exposure to all variables. In the post power assessment, the powers of $\mathrm{HC}(39.6 \%)$, anaemia (92.1\%) and betel nut chewing $(82.2 \%)$ were $<95 \%$, and the powers of the other variables were $>95 \%$.

The characteristics of the case and control groups in Groups I-IV are shown in Table 2, and the hierarchical generalised linear models were used to test the difference of proportion between cases and controls by controlling the hospital-clustering effects in each group (stratified analyses). Detailed information is shown in the supplementary file (univariate analyses Additional file 1: Table S1 and multivariate analyses Additional file 1: Table S2). Based on Additional file 1: Table S1 and Additional file 1: Table S2, Table 3 shows the pooled results of all variables.

Male $(p<0.001)$, hyperuricemia $(p<0.001)$, smoking $(p<0.001)$, lack of exercise habits $(p<0.001)$ and groundwater use $(p=0.003)$ were significant risk factors for CKD in the univariate analyses, and their heterogeneities were not significant. In the multivariate analyses, male 
Table 1 Overall proportion of cases and controls with exposure

\begin{tabular}{|c|c|c|c|c|}
\hline & & CKD & non-CKD & Post power \\
\hline \multirow[t]{2}{*}{ Gender } & Female & 2302 (43.2 \%) & 2943 (57.3 \%) & $>99.9 \%$ \\
\hline & Male & 3026 (56.8 \%) & 867 (42.7 \%) & \\
\hline Age & & $59.8 \pm 15.2$ & $54.0 \pm 15.1$ & $>99.9 \%$ \\
\hline \multirow[t]{2}{*}{ Obesity (151 missing) } & Normal & 4634 (88.7 \%) & 4650 (91.4 \%) & $>99.9 \%$ \\
\hline & Abnormal & $592(11.3 \%)$ & $436(8.6 \%)$ & \\
\hline \multirow[t]{3}{*}{ Income (61 missing) } & Low & 3056 (57.6 \%) & 2039 (40.0 \%) & \\
\hline & Median & 1416 (26.7 \%) & 1791 (35.1%) & $>99.9 \%$ \\
\hline & High & 832 (15.7 \%) & 1268 (24.9 \%) & $>99.9 \%$ \\
\hline \multirow[t]{2}{*}{ HB (1 missing) } & Normal & 5027 (94.4 \%) & 5064 (93.9 \%) & $99.4 \%$ \\
\hline & Abnormal & $300(5.6 \%)$ & $71(6.1 \%)$ & \\
\hline \multirow[t]{2}{*}{$\mathrm{HC}^{*}$ (2 missing) } & Normal & 5228 (98.2 \%) & 5064 (98.6 \%) & $39.6 \%$ \\
\hline & Abnormal & $98(1.8 \%)$ & $71(1.4 \%)$ & \\
\hline \multirow[t]{2}{*}{ Hyperuricaemia (2 missing) } & Normal & 4036 (75.8 \%) & 4852 (94.5 \%) & $98.7 \%$ \\
\hline & Abnormal & 1291 (24.2 \%) & $282(5.5 \%)$ & \\
\hline \multirow[t]{2}{*}{ Anaemia* (2 missing) } & Normal & 4463 (83.8 \%) & 4938 (96.2 \%) & $92.1 \%$ \\
\hline & Abnormal & $863(16.2 \%)$ & $197(3.8 \%)$ & \\
\hline \multirow[t]{2}{*}{ Hyperlipidaemia (1 missing) } & Normal & 3832 (71.9 \%) & 4196 (81.7 \%) & $>99.9 \%$ \\
\hline & Abnormal & 1495 (28.1%) & 939 (18.3 \%) & \\
\hline \multirow[t]{2}{*}{ Smoking status (338 missing) } & Never & 3909 (76.0 \%) & 4126 (82.9 \%) & $>99.9 \%$ \\
\hline & Ever & 1237 (24.0\%) & $853(17.1 \%)$ & \\
\hline \multirow[t]{2}{*}{ Alcohol intake (382 missing) } & Never & $4361(85.0 \%)$ & $4340(87.6 \%)$ & $>99.9 \%$ \\
\hline & Ever & $767(15.0 \%)$ & $613(12.4 \%)$ & \\
\hline \multirow[t]{2}{*}{ Betel nut chewing* (456 missing) } & Never & 4876 (95.7\%) & 4758 (96.9 \%) & $82.2 \%$ \\
\hline & Ever & $222(4.3 \%)$ & $151(3.1 \%)$ & \\
\hline \multirow[t]{2}{*}{ Exercise habits (173 missing) } & Never & 1862 (35.8 \%) & 1511 (29.7\%) & $>99.9 \%$ \\
\hline & Ever & 3338 (64.2\%) & 3579 (70.3 \%) & \\
\hline \multirow[t]{2}{*}{ Groundwater using (17 missing) } & Never & 5029 (94.5 \%) & 4890 (95.4 \%) & $96.6 \%$ \\
\hline & Ever & $293(5.5 \%)$ & $234(4.6 \%)$ & \\
\hline
\end{tabular}

CKD: patients with CKD; non-CKD: patients without CKD; HB: hepatitis $\mathrm{B}$; $\mathrm{HC}$ : hepatitis $\mathrm{C}$

$\S$ : Post power (1- $\beta$ ) estimate based on $G^{*}$ power [28]

Boldface $\&^{*}$ : the powers of each variable were less than $95 \%$, and they were defined as lacking in power

( $p<0.001)$, hyperuricemia $(p<0.001)$ and lack of exercise habits $(p<0.001)$ were significant and their heterogeneities were not significant. However, smoking $(p=0.026)$ and groundwater use $(p=0.022)$ were not significant after adjustment by the Bonferroni method. The pooled ORs of gender, hyperuricemia and exercise habits were 1.61 (95\% $\mathrm{CI}=1.45-1.78, \quad p<0.001), \quad 3.63 \quad(95 \% \mathrm{CI}=3.11-4.24$, $p<0.001)$ and $0.71(95 \% \mathrm{CI}=0.64-0.79, p<0.001)$. Age and income may relate to CKD, but they had high heterogeneity between the four groups ( $\mathrm{I}^{2}$ of age $85.7 \%, \mathrm{I}^{2}$ of income $80.8 \%$ and $82.3 \%$ ). This implies that the pooled ORs of these variables may be unreliable. However, the heterogeneities were significantly reduced after the adjusted demographic characteristics, the history of disease and lifestyle. The pooled ORs per 10 years of age was 1.44 (95\% CI $=1.13-1.83, p=0.003)$ and of income for Median vs. Low was $0.58(95 \% \mathrm{CI}=0.47-0.73 p<0.001)$ and for High vs. Low was 0.55 (95\% CI $=0.43-0.70, p<0.001$ ), which were all statistically significant.

The heterogeneities of obesity, HB, HC, alcohol intake and betel nut chewing were not significant in the univariate/multivariate analyses, and their pooled ORs were not significant. Based on the power of this study, obesity, $\mathrm{HB}$ and alcohol intake may not be associated with CKD (unless their true ORs were very small), but the power of the significance test in $\mathrm{HC}$ and betel nut chewing may be insufficient.

The results of anaemia and hyperlipidaemia were interesting. We found that there was high and significant heterogeneity among the four groups. The $\mathrm{I}^{2}$ of anaemia was $88.5 \%$ for univariate and $82.2 \%$ for multivariate analyses, and the $\mathrm{I}^{2}$ of hyperlipidaemia was $80.5 \%$ for 
Table 2 Characteristics of subjects by stratification for HTN and DM

\begin{tabular}{|c|c|c|c|c|c|c|c|c|c|}
\hline & & \multicolumn{2}{|l|}{ Group I } & \multicolumn{2}{|l|}{ Group II } & \multicolumn{2}{|l|}{ Group III } & \multicolumn{2}{|l|}{ Group IV } \\
\hline & & $\overline{C K D}$ & non-CKD & $\overline{C K D}$ & non-CKD & $\overline{C K D}$ & non-CKD & $\overline{C K D}$ & non-CKD \\
\hline \multirow[t]{2}{*}{ Gender } & Female & $670(48.5 \%)$ & $1536(63.9 \%)$ & $819(40.4 \%)$ & $752(51.2 \%)$ & $172(41.1 \%)$ & $304(50.4 \%)$ & $641(42.7 \%)$ & $351(53.3 \%)$ \\
\hline & Male & $712(51.5 \%)$ & $867(36.1 \%)$ & 1207 (59.6 \%) & $718(48.8 \%)$ & $247(58.9 \%)$ & $299(49.6 \%)$ & $860(57.3 \%)$ & 308 (46.7\%) \\
\hline Age & & $53.0 \pm 17.1$ & $47.3 \pm 15.2$ & $61.3 \pm 15.1$ & $60.5 \pm 13.0$ & $61.7 \pm 12.1$ & $57.6 \pm 12.1$ & $63.5 \pm 11.8$ & $60.8 \pm 10.8$ \\
\hline \multirow[t]{2}{*}{ Obesity (151 missing) } & Normal & $1278(93.6 \%)$ & $2234(94.0 \%)$ & 1787 (89.8 \%) & 1311 (90.1\%) & $356(86.2 \%)$ & $538(89.8 \%)$ & $1213(83.3 \%)$ & $567(86.6 \%)$ \\
\hline & Abnormal & $87(6.4 \%)$ & $143(6.0 \%)$ & $204(10.2 \%)$ & 144 (9.9 \%) & $57(13.8 \%)$ & $61(10.2 \%)$ & $244(16.7 \%)$ & 88 (13.4 \%) \\
\hline \multirow[t]{3}{*}{ Income (61 missing) } & Low & $648(47.3 \%)$ & $677(28.3 \%)$ & $1153(57.0 \%)$ & $736(50.3 \%)$ & $265(63.4 \%)$ & $260(44.0 \%)$ & $990(66.4 \%)$ & $366(56.2 \%)$ \\
\hline & Median & $434(31.7 \%)$ & $971(40.6 \%)$ & $554(27.4 \%)$ & 418 (28.6 \%) & 97 (23.2 \%) & $213(36.0 \%)$ & 331 (22.2 \%) & 189 (29.0\%) \\
\hline & High & $289(21.1 \%)$ & $746(31.2 \%)$ & $316(15.6 \%)$ & $308(21.1 \%)$ & $56(13.4 \%)$ & $118(20.0 \%)$ & $171(11.5 \%)$ & 96 (14.7\%) \\
\hline \multirow[t]{2}{*}{ HB (1 missing) } & Normal & $1273(92.1 \%)$ & $2222(92.5 \%)$ & 1905 (94.0\%) & 1399 (95.2 \%) & $402(95.9 \%)$ & $574(95.2 \%)$ & $1447(96.5 \%)$ & 629 (95.4\%) \\
\hline & Abnormal & 109 (7.9\%) & $181(7.5 \%)$ & $121(6.0 \%)$ & $71(4.8 \%)$ & $17(4.1 \%)$ & $29(4.8 \%)$ & $53(3.5 \%)$ & $30(4.6 \%)$ \\
\hline \multirow[t]{2}{*}{ HC (2 missing) } & Normal & $1358(98.3 \%)$ & $2377(98.9 \%)$ & 1984 (97.9\%) & 1446 (98.4 \%) & $412(98.3 \%)$ & $593(98.3 \%)$ & $1474(98.3 \%)$ & 648 (98.3\%) \\
\hline & Abnormal & $23(1.7 \%)$ & $26(1.1 \%)$ & $42(2.1 \%)$ & $24(1.6 \%)$ & $7(1.7 \%)$ & $10(1.7 \%)$ & $26(1.7 \%)$ & $11(1.7 \%)$ \\
\hline \multirow[t]{2}{*}{ Hyperuricemia (2 missing) } & Normal & $1185(85.7 \%)$ & $2335(97.2 \%)$ & 1398 (69.0 \%) & 1336 (90.9 \%) & $367(87.6 \%)$ & $572(94.9 \%)$ & $1086(72.4 \%)$ & $609(92.4 \%)$ \\
\hline & Abnormal & $197(14.3 \%)$ & $68(2.8 \%)$ & $628(31.0 \%)$ & $133(9.1 \%)$ & $52(12.4 \%)$ & $31(5.1 \%)$ & $414(27.6 \%)$ & $50(7.6 \%)$ \\
\hline \multirow[t]{2}{*}{ Anaemia (2 missing) } & Normal & $1217(88.1 \%)$ & $2285(95.1 \%)$ & $1674(82.6 \%)$ & 1431 (97.3\%) & $383(91.4 \%)$ & $588(97.5 \%)$ & $1189(79.3 \%)$ & 634 (96.2\%) \\
\hline & Abnormal & $165(11.9 \%)$ & $118(4.9 \%)$ & $352(17.4 \%)$ & $39(2.7 \%)$ & $36(8.6 \%)$ & $15(2.5 \%)$ & $310(20.7 \%)$ & $25(3.8 \%)$ \\
\hline \multirow[t]{2}{*}{ Hyperlipidaemia (1 missing) } & Normal & $1144(82.8 \%)$ & $2184(90.9 \%)$ & 1478 (73.0 \%) & $1123(76.4 \%)$ & $308(73.5 \%)$ & $460(76.3 \%)$ & $902(60.1 \%)$ & $429(65.1 \%)$ \\
\hline & Abnormal & $238(17.2 \%)$ & $219(9.1 \%)$ & $548(27.0 \%)$ & $347(23.6 \%)$ & $111(26.5 \%)$ & $143(23.7 \%)$ & $598(39.9 \%)$ & $230(34.9 \%)$ \\
\hline \multirow[t]{2}{*}{ Smoking status (338 missing) } & Never & $1088(80.5 \%)$ & $2041(87.6 \%)$ & 1496 (76.6 \%) & $1148(80.8 \%)$ & $288(71.1 \%)$ & $438(74.9 \%)$ & $1037(72.2 \%)$ & 499 (77.7\%) \\
\hline & Ever & $263(19.5 \%)$ & $290(12.4 \%)$ & 457 (23.4 \%) & $273(19.2 \%)$ & $117(28.9 \%)$ & $147(25.1 \%)$ & $400(27.8 \%)$ & $143(22.3 \%)$ \\
\hline \multirow[t]{2}{*}{ Alcohol intake (382 missing) } & Never & $1203(89.0 \%)$ & $2109(91.1 \%)$ & $1658(85.2 \%)$ & 1209 (85.4 \%) & $338(83.9 \%)$ & $480(82.2 \%)$ & $1162(81.4 \%)$ & $542(85.0 \%)$ \\
\hline & Ever & $148(11.0 \%)$ & $207(8.9 \%)$ & $289(14.8 \%)$ & $206(14.6 \%)$ & $65(16.1 \%)$ & $104(17.8 \%)$ & $265(18.6 \%)$ & $96(15.0 \%)$ \\
\hline \multirow[t]{2}{*}{ Betel nut chewing (456 missing) } & Never & $1296(96.7 \%)$ & $2257(98.5 \%)$ & 1870 (96.6 \%) & 1360 (97.0 \%) & $374(93.0 \%)$ & $539(92.8 \%)$ & $1336(94.1 \%)$ & $602(95.0 \%)$ \\
\hline & Ever & $44(3.3 \%)$ & $35(1.5 \%)$ & $66(3.4 \%)$ & $42(3.0 \%)$ & $28(7.0 \%)$ & $42(7.2 \%)$ & $84(5.9 \%)$ & $32(5.0 \%)$ \\
\hline \multirow[t]{2}{*}{ Exercise habits (173 missing) } & Never & $474(34.8 \%)$ & $755(31.7 \%)$ & $691(35.0 \%)$ & $389(26.7 \%)$ & $138(33.5 \%)$ & $173(28.9 \%)$ & $559(38.6 \%)$ & $194(29.6 \%)$ \\
\hline & Ever & $887(65.2 \%)$ & $1624(68.3 \%)$ & $1286(65.0 \%)$ & 1068 (73.3 \%) & $274(66.5 \%)$ & $426(71.1 \%)$ & $891(61.4 \%)$ & $461(70.4 \%)$ \\
\hline \multirow[t]{2}{*}{ Groundwater using (17 missing) } & Never & $1312(95.2 \%)$ & $2334(97.3 \%)$ & 1904 (94.0\%) & 1349 (92.0 \%) & $394(94.0 \%)$ & $574(95.5 \%)$ & $1419(94.7 \%)$ & $633(96.2 \%)$ \\
\hline & Ever & $66(4.8 \%)$ & $65(2.7 \%)$ & $122(6.0 \%)$ & $117(8.0 \%)$ & $25(6.0 \%)$ & $27(4.5 \%)$ & $80(5.3 \%)$ & $25(3.8 \%)$ \\
\hline
\end{tabular}

Group I: participants without DM and HTN; Group II: participants with HTN without DM; Group III: participants with DM without HTN; Group IV: participants with DM and HTN

CKD: patients with CKD; non-CKD: patients without CKD; HTN: hypertension; DM: diabetes mellitus; HB: hepatitis B; HC: hepatitis C 
Table 3 Pooled data showing effect of each risk factor on CKD and heterogeneity between the four groups§

\begin{tabular}{|c|c|c|c|c|c|c|}
\hline & \multicolumn{3}{|l|}{ Univariable analyses } & \multicolumn{3}{|l|}{ Multivariable analyses } \\
\hline & OR (95 \% Cl) & $1^{2}$ & Q test & OR $(95 \% \mathrm{Cl})$ & $1^{2}$ & $\overline{Q \text { test }}$ \\
\hline Gender (Male versus Female) & $1.65(1.49 \text { to } 1.82)^{*}$ & $25.5 \%$ & 0.296 & $1.61(1.45 \text { to } 1.78)^{*}$ & $0.0 \%$ & 0.934 \\
\hline Age (per 10 years) & $1.19(1.09 \text { to } 1.29)^{*} £$ & $85.7 \% *$ & $<0.001^{*}$ & $1.44(1.13 \text { to } 1.83)^{*}$ & $24.1 \%$ & 0.323 \\
\hline Obesity (Abnormal versus Normal) & 1.14 (0.93 to 1.40$)$ & $43.9 \%$ & 0.163 & 1.07 (0.86 to 1.33$)$ & $39.0 \%$ & 0.204 \\
\hline Income (Median versus Low) & $0.57(0.45 \text { to } 0.72)^{*} £$ & $80.8 \% *$ & $<0.001^{*}$ & $0.58(0.47 \text { to } 0.73)^{*}$ & $71.9 \%$ & 0.011 \\
\hline (High versus Low) & $0.50(0.38 \text { to } 0.67)^{*} £$ & $82.3 \% *$ & $<0.001^{*}$ & $0.55(0.43 \text { to } 0.70)^{*}$ & $65.0 \%$ & 0.022 \\
\hline HB (Abnormal versus Normal) & 1.08 (0.88 to 1.33$)$ & $21.6 \%$ & 0.263 & 1.25 (1.03 to 1.52$)$ & $0.0 \%$ & 0.523 \\
\hline HC (Abnormal versus Normal) & 1.29 (0.93 to 1.79$)$ & $0.0 \%$ & 0.707 & $1.22(0.85$ to 1.74$)$ & $0.0 \%$ & 0.778 \\
\hline Hyperuricaemia (Abnormal versus Normal) & $4.56(3.96 \text { to } 5.26)^{*}$ & $0.0 \%$ & 0.213 & $3.63(3.11 \text { to } 4.24)^{*}$ & $0.0 \%$ & 0.806 \\
\hline Anaemia (Abnormal versus Normal) & $4.89(2.76 \text { to } 8.66)^{*} £$ & $88.5 \% *$ & $<0.001^{*}$ & $4.64(2.81 \text { to } 7.65)^{*} £$ & $82.2 \% *$ & $<0.001^{*}$ \\
\hline Hyperlipidaemia (Abnormal versus Normal) & $1.48(1.17 \text { to } 1.88)^{*} £$ & $80.5 \% *$ & 0.001 & $1.28(0.97$ to 1.67$) £$ & $81.3 \% *$ & $0.001^{*}$ \\
\hline Smoking status (Abnormal versus Normal) & $1.45(1.28 \text { to } 1.64)^{*}$ & $26.6 \%$ & 0.305 & $1.17(1.02$ to 1.34$)$ & $0.0 \%$ & 0.559 \\
\hline Alcohol intake (Abnormal versus Normal) & $1.15(1.00$ to 1.33$)$ & $22.3 \%$ & 0.248 & 0.83 (0.71 to 0.97$)$ & $0.0 \%$ & 0.701 \\
\hline Betel nut chewing (Abnormal versus Normal) & $1.35(1.03$ to 1.77$)$ & $29.0 \%$ & 0.233 & 1.10 (0.85 to 1.44$)$ & $0.0 \%$ & 0.836 \\
\hline Exercise habits (Abnormal versus Normal) & $0.74(0.65 \text { to } 0.85)^{*}$ & $50.9 \%$ & 0.106 & $0.71(0.64 \text { to } 0.79)^{*}$ & $0.0 \%$ & 0.859 \\
\hline Groundwater using (Abnormal versus Normal) & $1.44(1.13$ to 1.83$)$ & $24.1 \%$ & 0.323 & $1.29(1.04$ to 1.61$)$ & $0.0 \%$ & 0.534 \\
\hline
\end{tabular}

§: The four groups were Group I (participants without DM and HTN), Group II (participants with HTN without DM), Group III (participants with DM without HTN) and Group IV (participants with DM and HTN)

HB: hepatitis B; HC: hepatitis C

OR: pooled odds ratio for variation groups compared with reference groups on CKD; $95 \% \mathrm{Cl}: 95 \%$ confidence interval of OR

$I^{2}$ : heterogeneity between four groups in each variable; $Q$ test: the significant test of $I^{2}$ using Cochrane $Q$ test

Boldface $\& *^{*}$ : significance after Bonferroni adjustment: $p$ value $<0.05 / 14=0.0036$

$\mathrm{f}:$ The pooled results were unreliable because the difference between coefficients in four group were significant. Please refer to the results of stratified analyses in Supplementary File (univariable analyses: Additional file 1: Table S1; multivariable analyses: Additional file 1: Table S2)

univariate and $81.3 \%$ for multivariate analyses. These results imply that the pooled ORs may be unreliable. Fig. 2 shows further analyses that indicate the causes of heterogeneity for anaemia $(93.7 \%)$ and hyperlipidaemia (100 \%) may be due to HTN. After the recombined analyses, the ORs of anaemia and hyperlipidaemia were 6.75 (95\% CI $=4.76-9.68, p<0.001)$ and $1.03(95 \% \mathrm{CI}=$ $0.89-1.19, p=0.695)$, respectively, in patients with HTN, and the ORs of anaemia and hyperlipidaemia were 2.83 (95\% CI $=2.16-3.67, p<0.001)$ and $1.67(95 \% \mathrm{CI}=$ $1.38-2.01, p<0.001)$, respectively, in people without HTN. These results represent an additive interaction effect of anaemia and HTN on CKD, and an antagonistic interaction effect of hyperlipidaemia and HTN on CKD.

\section{Discussion}

The results of this study found that male, ageing, low income, hyperuricemia and lack of exercise habits were risk factors for CKD. The effects of anaemia and hyperlipidaemia on CKD in patients with or without HTN were different. We also found that $\mathrm{HB}, \mathrm{HC}$, smoking, alcohol intake, betel nut chewing and groundwater use may not be associated with CKD. Among males, ageing and lack of exercise habits were the traditional risk factors for CKD $[7,6,17,16]$. In our study, we also observed a significant association between these risk factors and CKD, and we did not find high heterogeneity among the four groups. The discussion that follows divides the associations between other variables and CKD into three parts: socioeconomic status-related factors, possible reasons for the high prevalence of ESRD in Taiwan and the interactive effects between anaemia/hyperlipidaemia and HTN on CKD.

Low income was an important predictive factor for CKD, and it was characteristic of people with a low socioeconomic status. Socioeconomic status may be related to many risk factors, such as second-hand tobacco smoke [30] and unhealthy diets [31]. In this study, we investigated some socioeconomic status-related factors (smoking status [32], alcohol intake [33], betel nut chewing [34] and groundwater use [18]), and we found that the association between smoking status/groundwater use and CKD may be related to income level. Their effects were significant before the adjustment of income but not after this adjustment.

The ORs of alcohol intake and betel nut chewing in the univariate and multivariate analyses also presented a similar phenomenon. Previous studies that had investigated the above factors did not adjust for socioeconomic status. Thus, they may have overrated their risk on CKD [14, 15, 18]. Based on the power of this study, further research is needed concerning the association between betel nut chewing and CKD. With smoking status, alcohol intake 


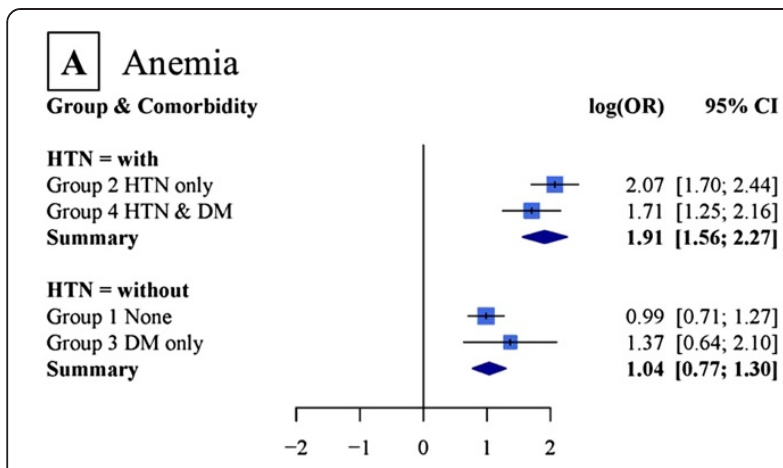

B Hyperlipidemia

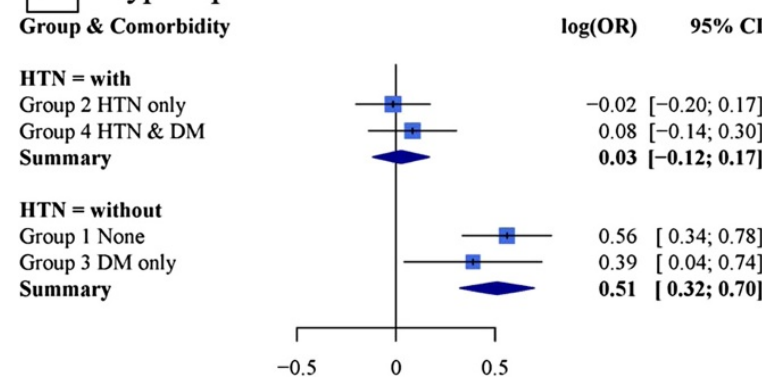

Fig. 2 Stratified analyses for variables with significant heterogeneity between the four groups using multivariable analyses. (a) shows coefficients of anaemia and (b) shows coefficients of

hyperlipidaemia using multivariate analyses and their heterogeneity caused by HTN in the different groups. Amount of heterogeneity accounted for by HTN was $93.71 \% / 100 \%$. The odds ratios and $95 \%$ $\mathrm{Cl}$ of anaemia in patients with or without HTN were 6.75 (4.76-9.68) and 2.83 (2.16-3.67), respectively. The odds ratios $(95 \% \mathrm{Cl}$ ) of hyperlipidaemia in patients with or without HTN were 1.03 (0.89-1.19) and 1.67 (1.38-2.01), respectively HTN: hypertension; DM: diabetes mellitus; log (OR): natural logarithm of the odds ratio; $95 \%$ Cl: $95 \%$ confidence interval of log (OR)

and groundwater use, these may have a smaller impact on CKD. Intervention for these small impact factors may not be effective, and therefore, we determined that smoking status, alcohol intake and betel nut chewing were not the best targets for CKD prevention.

The prevalence of HB [35] and HC [36] in Taiwan is higher than in most other countries, and Taiwan has the highest prevalence and third highest incidence of ESRD in the world [2]. Previous studies have reported the association between HC and CKD [11], so we suspected that the high prevalence of hepatitis might be the main reason for the high prevalence of ESRD. However, our study showed the nonsignificance of these factors. Therefore, we determined that $\mathrm{HB}$ and $\mathrm{HC}$ were not the main reason for the high prevalence and incidence of ESRD in Taiwan, but the power of $\mathrm{HC}$ may be insufficient and needs further research.

People with hyperuricemia have a higher risk of ESRD than those without it, and the association was found to be very high in this study. Despite the lack of detailed statistics worldwide, we believe that the prevalence of hyperuricemia in Taiwan may be higher than in many other countries [37, 38], and this may also play a key role in ESRD in Taiwan. However, our study might have overrated the risk of hyperuricemia in CKD. Previous studies presented a significant association between hyperuricemia and CKD, but the relative risks in these studies were $<2[7,21]$. Therefore, we determined that hyperuricemia might be associated with CKD, but the effect of intervention for hyperuricemia-related factors on CKD might not be very effective.

Anaemia is a likely complication in patients with CKD, but this is not a cause [39]. Therefore, it is not a good target for the prevention of CKD. However, this study was not only concerned with prevention strategies but also with screening strategies for CKD. Previous studies have demonstrated that awareness of CKD in patients is very low $[40,41]$. Therefore, physicians might have to help them with monitoring the CKD. This study showed a strong association between anaemia and CKD in patients with HTN but not in the general population. This result helps to better pinpoint CKD high risk groups. Patients with both anaemia and HTN are a newly discovered high risk group for CKD, and physicians in outpatient clinics need to recognise that patients with anaemia accompanied with HTN might be latent CKD cases.

The risk effect of hyperlipidaemia on CKD was only found to be in the general population, but not in patients with HTN as reported in an earlier study [7]. Male subjects had a higher prevalence of HTN than females, and the hazard ratio of hyperlipidaemia on CKD in males was lower than in females [7]. Obesity was a hyperlipidaemiarelated factor, and the risk effects of obesity presented similar results (Additional file 1: Table S1 and Additional file 1: Table S2). Although the results were not significant, we still found that the ORs in patients with HTN were lower than those in people without HTN. In addition, the heterogeneity of obesity was $39.0 \%$ in the multivariate analysis, which implies that HTN might be a moderate factor in the association between obesity-related factors and CKD. It has been reported that renal lipid accumulation is nephrotoxic and could play a role in CKD [42], but we are unable to explain why the association between hyperlipidaemia and CKD in patients with HTN has a negative impact. Further research is required to understand the basic mechanism.

This study has two limitations. First, a cross-sectional study is not the way to distinguish whether correlations were causative or not, and time relationships were not confirmed in this study. Most of our discussion has focused on this issue, and we surmised that associations between some factors and CKD might not be causative. Although these may not help prevent CKD, they are useful for planning a screening strategy. Low awareness 
of CKD is an important issue, and a good outpatient screening strategy is urgently needed. Second, the risk factors assessment was based on a structured questionnaire rather than on the laboratory data. This may have caused some misclassification. However, Taiwan has good medical accessibility and most people can easily obtain medical resources and understand their disease status. In addition, our interviewers were highly trained, and we regularly held meetings for feedback from the interviewers. This ensured the quality of our research and reduced the possibility of misclassification.

\section{Conclusions}

Gender, age, income, hyperuricemia, anaemia, hyperlipidaemia and exercise habits were good targets for planning a screening/prevention strategy for CKD in healthy populations and in patients with DM. In addition, the important evidence from this study confirmed that $\mathrm{HB}$, smoking status, alcohol intake and groundwater use were not good targets for CKD prevention. They were not associated with CKD or only had a low impact on the condition. We believe that intervention for related factors might not be an efficient method based on the strong statistical power in this study. The associations between $\mathrm{HC} /$ betel nut chewing and CKD require further research because they were underpowered in this study. Finally, this study suggested that a specific CKD screening/prevention strategy for patients with DM might not be efficient without laboratory data analyses, and the strategy for the general population could be used in patients with DM. Furthermore, we determined that a screening/prevention strategy for CKD in patients with HTN might differ from that of a healthy population. Hyperlipidaemia-related factors might not be a good target for patients with HTN, and physicians need to recognise that patients with HTN in anaemia outpatient clinics might be potential CKD patients. In addition, there is a need for better care in patients with anaemia and HTN and timely intervention is required when there are signs of deterioration.

\section{Additional file}

Additional file 1: Table S1. Effect of risk factors on CKD by hierarchical generalised linear models. Table S2: Effect of risk factors on CKD after adjusted demographic characteristics and history of disease and lifestyle.

\begin{abstract}
Abbreviations
BMI: Body mass index; Cl: Confidence interval; CKD: Chronic kidney disease; NTD: New Taiwan dollars; DM: Diabetes mellitus; eGFR: Estimated glomerular filtration rate; ESRD: End-stage renal disease; HTN: Hypertension; HB: hepatitis B; HC: hepatitis C; OR: Odds ratio; SD: Standard deviation.
\end{abstract}

\section{Competing interests}

The authors declare that they have no competing interests.

\section{Authors' contributions}

Conceived and designed the experiments: SLS CL HYY CCW HYC YFL. Performed the experiments: SLS CL HYY CCW JSC. Analysed the data: SLS CL SYK YLC JSC FCS MYW HYC. Contributed reagents/materials/analysis tools: KCL CHL YCK CTL YY CWY SJH MCW YHH YMH MYW. Wrote the paper: SLS CL HYY HYC YFL. All authors read and approved the final manuscript.

\section{Acknowledgement}

This study was supported by grants from the Health Promotion Administration, Ministry of Health and Welfare, Institute for Biotechnology and Medicine Industry, Taiwan, ROC (DOH97-HP-1101, DOH-98-1110, DOH99-HP-1106, DOH100-HP-1102).

\section{Author details}

${ }^{1}$ School of Public Health, National Defense Medical Center, Taipei, Taiwan. ${ }^{2}$ Graduate Institute of Life Sciences, National Defense Medical Center, Taipei, Taiwan. ${ }^{3}$ Division of Nephrology, Department of Medicine, Tri-Service General Hospital, National Defense Medical Center, Taipei, Taiwan. ${ }^{4}$ Division of Nephrology, Department of Medicine, Cardinal Tien Hospital, School of Medicine, Fu Jen Catholic University, New Taipei City, Taiwan. ${ }^{5}$ School of Public Health, Graduate Institute of Clinical Medical Science, China Medical University, Taichung, Taiwan. ${ }^{6}$ Division of Nephrology, Kaohsiung Chang Gung Memorial Hospital, Chang Gung Medical University, Kaohsiung, Taiwan. ${ }^{7}$ The Division of Nephrology, Changhua Christian Hospital, Changhua, Taiwan. ${ }^{8}$ School of Medicine, Chang Gung University, Taoyuan, Taiwan. ${ }^{9}$ Division of Nephrology, Department of Medicine, Kaohsiung Medical University Hospital, Kaohsiung, Taiwan. ${ }^{10}$ Department of internal Medicine, Cheng Kung University Medicial Center, Tainan, Taiwan. ${ }^{11}$ Division of Nephrology, Department of Medicine, Shuang Ho Hospital, Graduate Institute of Clinical Medicine, Taipei Medical University, No. 250, Wuxing St., Xinyi District, Taipei 110, Taiwan. ${ }^{12} \mathrm{~S}$ chool of Public Health, Taipei Medical University, No. 250, Wuxing St., Xinyi District, Taipei 110, Taiwan.

Received: 14 October 2014 Accepted: 19 May 2015

Published online: 16 June 2015

\section{References}

1. Kuo HW, Tsai SS, Tiao MM, Yang CY. Epidemiological features of CKD in Taiwan. American journal of kidney diseases : the official journal of the National Kidney Foundation. 2007;49(1):46-55. doi:10.1053/j.ajkd.2006.10.007.

2. Bethesda MD. Annual Data Report: Atlas of Chronic Kidney Disease and End-Stage Renal Disease in the United States. U.S. Renal Data System, USRDS, National Institutes of Health, National Institute of Diabetes and Digestive and Kidney Diseases. 2013. http://www.usrds.org/2013/pdf/ V2_ch12_13.pdf. Accessed November 122013.

3. Go AS, Chertow GM, Fan D, McCulloch CE, Hsu CY. Chronic kidney disease and the risks of death, cardiovascular events, and hospitalization. The New England journal of medicine. 2004;351(13):1296-305. doi:10.1056/ NEJMoa041031.

4. Li PK, Weening JJ, Dirks J, Lui SL, Szeto CC, Tang S et al. A report with consensus statements of the International Society of Nephrology 2004 Consensus Workshop on Prevention of Progression of Renal Disease, Hong Kong, June 29, 2004. Kidney international Supplement. 2005(94):S2-7. doi:10.1111/j.1523-1755.2005.09401.x.

5. Hallan SI, Dahl K, Oien CM, Grootendorst DC, Aasberg A, Holmen J et al. Screening strategies for chronic kidney disease in the general population: follow-up of cross sectional health survey. BMJ (Clinical research ed). 2006;333(7577):1047. doi:10.1136/bmj.39001.657755.BE.

6. Neugarten J, Acharya A, Silbiger SR. Effect of gender on the progression of nondiabetic renal disease: a meta-analysis. Journal of the American Society of Nephrology : JASN. 2000;11(2):319-29.

7. Yamagata K, Ishida K, Sairenchi T, Takahashi H, Ohba S, Shiigai T, et al. Risk factors for chronic kidney disease in a community-based population: a 10-year follow-up study. Kidney international. 2007;71(2):159-66. doi:10.1038/sj.ki.5002017.

8. Ramkumar N, Cheung AK, Pappas LM, Roberts WL, Beddhu S. Association of obesity with inflammation in chronic kidney disease: a cross-sectional study. Journal of renal nutrition : the official journal of the Council on Renal Nutrition of the National Kidney Foundation. 2004;14(4):201-7.

9. Fored CM, Ejerblad E, Fryzek JP, Lambe M, Lindblad P, Nyren O, et al. Socio-economic status and chronic renal failure: a population-based 
case-control study in Sweden. Nephrology, dialysis, transplantation: official publication of the European Dialysis and Transplant Association - European Renal Association. 2003;18(1):82-8.

10. Ayodele OE, Salako BL, Kadiri S, Arije A, Alebiosu CO. Hepatitis B virus infection: implications in chronic kidney disease, dialysis and transplantation. African journal of medicine and medical sciences. 2006;35(2):111-9.

11. Sabry AA, Sobh MA, Irving WL, Grabowska A, Wagner BE, Fox S, et al. A comprehensive study of the association between hepatitis $C$ virus and glomerulopathy. Nephrology, dialysis, transplantation : official publication of the European Dialysis and Transplant Association - European Renal Association. 2002;17(2):239-45.

12. Mehdi U, Toto RD. Anemia, diabetes, and chronic kidney disease. Diabetes care. 2009;32(7):1320-6. doi:10.2337/dc08-0779.

13. Orth SR. Smoking and the kidney. Journal of the American Society of Nephrology : JASN. 2002;13(6):1663-72.

14. Perneger TV, Whelton PK, Puddey IB, Klag MJ. Risk of end-stage renal disease associated with alcohol consumption. American journal of epidemiology. 1999;150(12):1275-81.

15. Kang IM, Chou CY, Tseng YH, Huang CC, Ho WY, Shih CM, et al. Association between betelnut chewing and chronic kidney disease in adults. Journal of occupational and environmental medicine/American College of Occupational and Environmental Medicine. 2007;49(7):776-9. doi:10.1097/JOM.0b013e318095a48a.

16. Eidemak I, Haaber AB, Feldt-Rasmussen B, Kanstrup IL, Strandgaard S. Exercise training and the progression of chronic renal failure. Nephron. 1997;75(1):36-40.

17. Leehey DJ, Moinuddin I, Bast JP, Qureshi S, Jelinek CS, Cooper C, et al. Aerobic exercise in obese diabetic patients with chronic kidney disease: a randomized and controlled pilot study. Cardiovascular diabetology. 2009;8:62. doi:10.1186/1475-2840-8-62

18. Chandrajith R, Dissanayake CB, Ariyarathna T, Herath HM, Padmasiri JP. Dose-dependent $\mathrm{Na}$ and $\mathrm{Ca}$ in fluoride-rich drinking water-another major cause of chronic renal failure in tropical arid regions. The Science of the total environment. 2011;409(4):671-5. doi:10.1016/j.scitotenv.2010.10.046

19. Tseng $\mathrm{CH}$, Chong CK, Tseng CP, Tai TY. The association between urinary albumin excretion and ankle-brachial index in elderly Taiwanese patients with type 2 diabetes mellitus. Age and ageing. 2008;37(1):77-82 doi:10.1093/ageing/afm148.

20. Stengel B, Couchoud C, Cenee S, Hemon D. Age, blood pressure and smoking effects on chronic renal failure in primary glomerular nephropathies. Kidney international. 2000;57(6):2519-26. doi:10.1046/j.1523-1755.2000.00111.x.

21. Liu WC, Hung CC, Chen SC, Yeh SM, Lin MY, Chiu YW, et al. Association of hyperuricemia with renal outcomes, cardiovascular disease, and mortality. Clinical journal of the American Society of Nephrology : CJASN. 2012;7(4):541-8. doi:10.2215/cjn.09420911.

22. Levey AS, Bosch JP, Lewis JB, Greene T, Rogers N, Roth D. A more accurate method to estimate glomerular filtration rate from serum creatinine: a new prediction equation. Modification of Diet in Renal Disease Study Group Annals of internal medicine. 1999;130(6):461-70.

23. Alberti KG, Zimmet PZ. Definition, diagnosis and classification of diabetes mellitus and its complications. Part 1: diagnosis and classification of diabetes mellitus provisional report of a WHO consultation. Diabetic medicine: a journal of the British Diabetic Association. 1998;15(7):539-53. doi:10.1002/(sici)1096-9136(199807)15:7<539::aid-dia668>3.0.co;2-s

24. De Backer G, Ambrosioni E, Borch-Johnsen K, Brotons C, Cifkova R, Dallongeville $J$, et al. European guidelines on cardiovascular disease prevention in clinical practice. Third Joint Task Force of European and Other Societies on Cardiovascular Disease Prevention in Clinical Practice Eur Heart J. 2003;24(17):1601-10.

25. Patwardhan VN. Nutritional anemias-WHO research program. Early developments and progress report of collaborative studies The American journal of clinical nutrition. 1966;19(1):63-71.

26. Zhang Y, Post WS, Dalal D, Blasco-Colmenares E, Tomaselli GF, Guallar E. Coffee, alcohol, smoking, physical activity and QT interval duration: results from the Third National Health and Nutrition Examination Survey. PloS one. 2011;6(2), e17584. doi:10.1371/journal.pone.0017584.

27. Burger M, Mensink G, Bronstrup A, Thierfelder W, Pietrzik K. Alcohol consumption and its relation to cardiovascular risk factors in Germany. European journal of clinical nutrition. 2004;58(4):605-14. doi:10.1038/ sj.ejcn.1601854.
28. Faul F, Erdfelder E, Buchner A, Lang AG. Statistical power analyses using G*Power 3.1: tests for correlation and regression analyses. Behavior research methods. 2009;41(4):1149-60. doi:10.3758/brm.41.4.1149.

29. Higgins JP, Thompson SG, Deeks JJ, Altman DG. Measuring inconsistency in meta-analyses. BMJ (Clinical research ed). 2003;327(7414):557-60. doi:10.1136/bmj.327.7414.557.

30. Bonevski B, Paul C, Jones A, Bisquera A, Regan T. Smoky homes: Gender, socioeconomic and housing disparities in second hand tobacco smoke (SHS) exposure in a large population-based Australian cohort. Preventive medicine. 2013. doi:10.1016/j.ypmed.2013.12.024.

31. Pilic L, Dzakula A. Socioeconomic status and risky health behaviors in Croatian adult population. Acta medica Croatica : casopis Hravatske akademije medicinskih znanosti. 2013;67(1):25-35.

32. Schori D, Hofmann $K$, Abel T. Social inequality and smoking in young Swiss men: intergenerational transmission of cultural capital and health orientation. International journal of public health. 2013. doi:10.1007/s00038-013-0537-3.

33. Giang KB, Van Minh H, Allebeck P. Alcohol consumption and household expenditure on alcohol in a rural district in Vietnam. Global health action. 2013;6:18937. doi:10.3402/gha.v6i0.18937.

34. Huang HL, Lee $\mathrm{CH}$, Yen YY, Chen T, Chen FL, Ho PS, et al. School-level contextual factors associated with betel quid chewing among schoolchildren in Taiwan. Community dentistry and oral epidemiology. 2009;37(1):58-67. doi:10.1111/j.1600-0528.2008.00442.x.

35. Hennessey K, Mendoza-Aldana J, Bayutas B, Lorenzo-Mariano KM, Diorditsa S. Hepatitis B control in the World Health Organization's Western Pacific Region: Targets, strategies, status. Vaccine. 2013;31 Suppl 9:J85-92. doi:10.1016/j.vaccine.2012.10.082.

36. Mohd Hanafiah K, Groeger J, Flaxman AD, Wiersma ST. Global epidemiology of hepatitis C virus infection: new estimates of age-specific antibody to HCV seroprevalence. Hepatology (Baltimore, Md). 2013;57(4):1333-42. doi:10.1002/hep.26141

37. Lee MS, Lin SC, Chang HY, Lyu LC, Tsai KS, Pan WH. High prevalence of hyperuricemia in elderly Taiwanese. Asia Pacific journal of clinical nutrition. 2005;14(3):285-92.

38. $B L, T W, H n Z, W w Y, H p Y, C x L$ et al. The prevalence of hyperuricemia in China: a meta-analysis. BMC public health. 2011;11:832. doi:10.1186/14712458-11-832.

39. Annear NM, Banerjee D, Joseph J, Harries TH, Rahman S, Eastwood JB. Prevalence of chronic kidney disease stages 3-5 among acute medica admissions: another opportunity for screening. QJM : monthly journal of the Association of Physicians. 2008;101(2):91-7. doi:10.1093/qjmed/hcm130.

40. Flessner MF, Wyatt SB, Akylbekova EL, Coady S, Fulop T, Lee F, et al. Prevalence and awareness of CKD among African Americans: the Jackson Heart Study. American journal of kidney diseases : the official journal of the National Kidney Foundation. 2009;53(2):238-47. doi:10.1053/j.ajkd.2008.08.035.

41. Tuot DS, Plantinga LC, Hsu CY, Jordan R, Burrows NR, Hedgeman E, et al. Chronic kidney disease awareness among individuals with clinical markers of kidney dysfunction. Clinical journal of the American Society of Nephrology : CJASN. 2011;6(8):1838-44. doi:10.2215/cjn.00730111.

42. Wahba IM, Mak RH. Obesity and obesity-initiated metabolic syndrome: mechanistic links to chronic kidney disease. Clinical journal of the American Society of Nephrology : CJASN. 2007;2(3):550-62. doi:10.2215/cjn.04071206.

\section{Submit your next manuscript to BioMed Central and take full advantage of:}

- Convenient online submission

- Thorough peer review

- No space constraints or color figure charges

- Immediate publication on acceptance

- Inclusion in PubMed, CAS, Scopus and Google Scholar

- Research which is freely available for redistribution 